\title{
KH in Paraffin: A Useful Organic Base for Organic Synthesis
}

\author{
Douglass F. Taber* and Christopher G. Nelson
}

Department of Chemistry and Biochemistry, University of Delaware, Newark, DE 19716

\section{Supporting Information}

\section{Contents:}

S-1 Table of Contents

S-2 General Experimental

S-3 Characterization of alkenes $\mathbf{8 , 9}$ and $\mathbf{1 0}$

S-4 $\quad{ }^{1} \mathrm{H}$ and ${ }^{13} \mathrm{C}$ NMR of alkene 7

S-5 $\quad{ }^{1} \mathrm{H}$ and ${ }^{13} \mathrm{C}$ NMR of alkene 8

S-6 $\quad{ }^{1} \mathrm{H}$ and ${ }^{13} \mathrm{C}$ NMR of alkene 9

S-7 $\quad{ }^{1} \mathrm{H}$ and ${ }^{13} \mathrm{C}$ NMR of alkene $\mathbf{1 0}$

S-8 $\quad{ }^{1} \mathrm{H}$ and ${ }^{13} \mathrm{C}$ NMR of alkene $\mathbf{1 1}$ 


\section{Experimental Section}

General. All reagents were of commercial quality. THF was freshly distilled under nitrogen from sodium/benzophenone before use. ${ }^{1} \mathrm{H}$ and ${ }^{13} \mathrm{C}$ NMR spectra were recorded in $\mathrm{CDCl}_{3}$ at $400 \mathrm{MHz}$ using TMS as the internal standard. ${ }^{13} \mathrm{C}$ multiplicities were determined with the aid of a JVERT pulse sequence, differentiating the signals for methyl and methine carbons as "d", from methylene and quaternary carbons as "u". IR spectra were measured as thin films. All reactions were run under an $\mathrm{N}_{2}$ atmosphere. TLC solvent mixtures are volume/volume. MTBE is methyl $t$-butyl ether and PE is petroleum ether. 


\title{
Alkene 8
}

Yield; $111 \mathrm{mg}, 0.48 \mathrm{mmol}, 48 \%$; $\mathrm{TLC} \mathrm{R} \mathrm{R}_{f}(\mathrm{MTBE} /$ petroleum ether $=2: 3)=0.81 ;{ }^{1} \mathrm{H}$ NMR $\delta 0.91(\mathrm{t}, \mathrm{J}=7.3 \mathrm{~Hz}, 3 \mathrm{H}), 1.40(\mathrm{~m}, \mathrm{~J}=7.4 \mathrm{~Hz}, 2 \mathrm{H}), 2.06$ (q, J=7.4 Hz, 2H), 2.50 (q, $\mathrm{J}=8.2 \mathrm{~Hz}, 2 \mathrm{H}), 3.50$ (t, J=7.0 Hz, 2H), 4.52 (s, 2H), 5.33 (dt, J=10.5, 7.5 Hz, 1H), 5.70

$(\mathrm{m}, 7.5 \mathrm{~Hz}, 1 \mathrm{H}), 6.03(\mathrm{t}, \mathrm{J}=10.5 \mathrm{~Hz}, 1 \mathrm{H}), 6.27(\mathrm{t}, \mathrm{J}=13.5 \mathrm{~Hz}, 1 \mathrm{H}), 7.1-7.4(\mathrm{~m}, 5 \mathrm{H}) ;{ }^{13} \mathrm{C}$ NMR $\delta$ d 13.8, 125.3, 125.6, 127.5, 127.7, 128.4, 130.4, 135.4; u 22.5, 28.5, 35.0, 138.6; IR 2958, 1722, $1102 \mathrm{~cm}^{-1}$; MS m/z 230 (6), 160 (13), 146 (21), 124 (10), 109 (100); HRMS calcd for $\mathrm{C}_{16} \mathrm{H}_{22} \mathrm{O} 230.1671$, obsd 230.1661.

\begin{abstract}
Alkene 9
Yield; $185 \mathrm{mg}, 0.90 \mathrm{mmol}, 91 \%$; $\mathrm{TLC} \mathrm{R} \mathrm{R}_{f}(\mathrm{MTBE} /$ petroleum ether $=2: 3)=0.80 ;{ }^{1} \mathrm{H}$ NMR $\delta 0.94$ (d, J=6.8 Hz, 6H), 2.38 (q, J=6.9 Hz, 2H), 2.60 (m, J=6.9 Hz, 1H), 3.47 (t, $\mathrm{J}=7.0 \mathrm{~Hz}, 2 \mathrm{H}), 4.51$ (s, 2H), 5.27 (m, 2H), 7.21-7.33 (m, 5H); ${ }^{13} \mathrm{C}$ NMR $\delta \mathrm{d} 23.2,26.7$, 123.1, 127.5, 127.7, 128.4, 139.6; u 26.7, 70.2, 72.9, 138.6; IR 2957, 1590, $1100 \mathrm{~cm}^{-1}$; MS m/z 107 (100), 113 (76), 131 (16), 161 (35), 204 (3) ); HRMS calcd for $\mathrm{C}_{14} \mathrm{H}_{20} \mathrm{O}$ 204.1514, obsd 204.1512.
\end{abstract}

\footnotetext{
Alkene 10

Yield; $88 \mathrm{mg}, 0.66 \mathrm{mmol}, 66 \%$; TLC R $(\mathrm{MTBE} /$ petroleum ether $=1: 10)=0.50 ;{ }^{1} \mathrm{H}$ NMR $\delta 3.80$ (s, 3H), 5.24 (d, J=10.8 Hz, 1H), 5.73 (d, J=17.5 Hz, 1H), 6.68 (dd, J=17.5, $10.8 \mathrm{~Hz}, 1 \mathrm{H}), 6.80$ (dd, J=8.3, 2.7 Hz, 1H), 6.94, (s, 1H), 7.00 (d, J=7.7 Hz, 1H), 7.23 (t, $\mathrm{J}=7.9 \mathrm{~Hz}, 1 \mathrm{H}) ;{ }^{13} \mathrm{C}$ NMR $\delta \mathrm{d} 55.2,111.6,113.5,118.9$, 129.5, 136.8; u 114.1, 139.1, 159.9; IR 3005, 1599, $1110 \mathrm{~cm}^{-1}$; MS m/z 134 (100), 119 (7), 104 (21); HRMS calcd for $\mathrm{C}_{9} \mathrm{H}_{10} \mathrm{O}$ 134.0732, obsd 134.0733.
} 
${ }^{1}$ H NMR Spectrum of Alkene 7
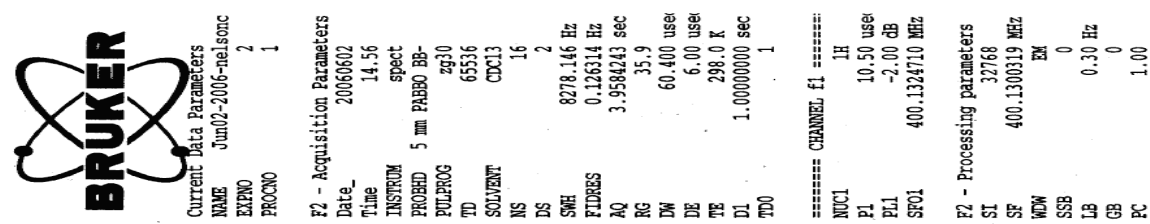

$9 z \cdot T$

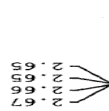

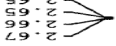

$95 \cdot \varepsilon-$

os.

${ }_{\varepsilon L}^{O L}: \frac{S}{S}=$

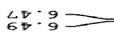

统:

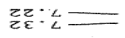

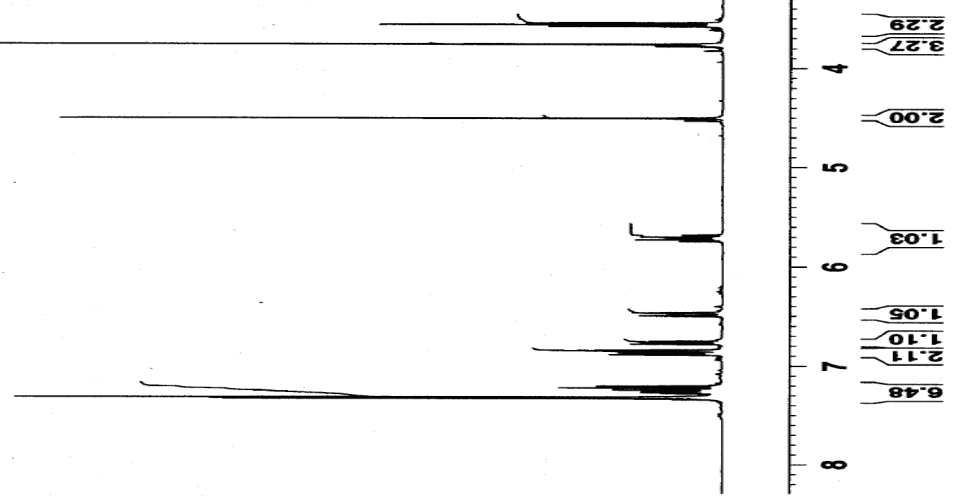

${ }^{13} \mathrm{C}$ Spectrum of Alkene 7
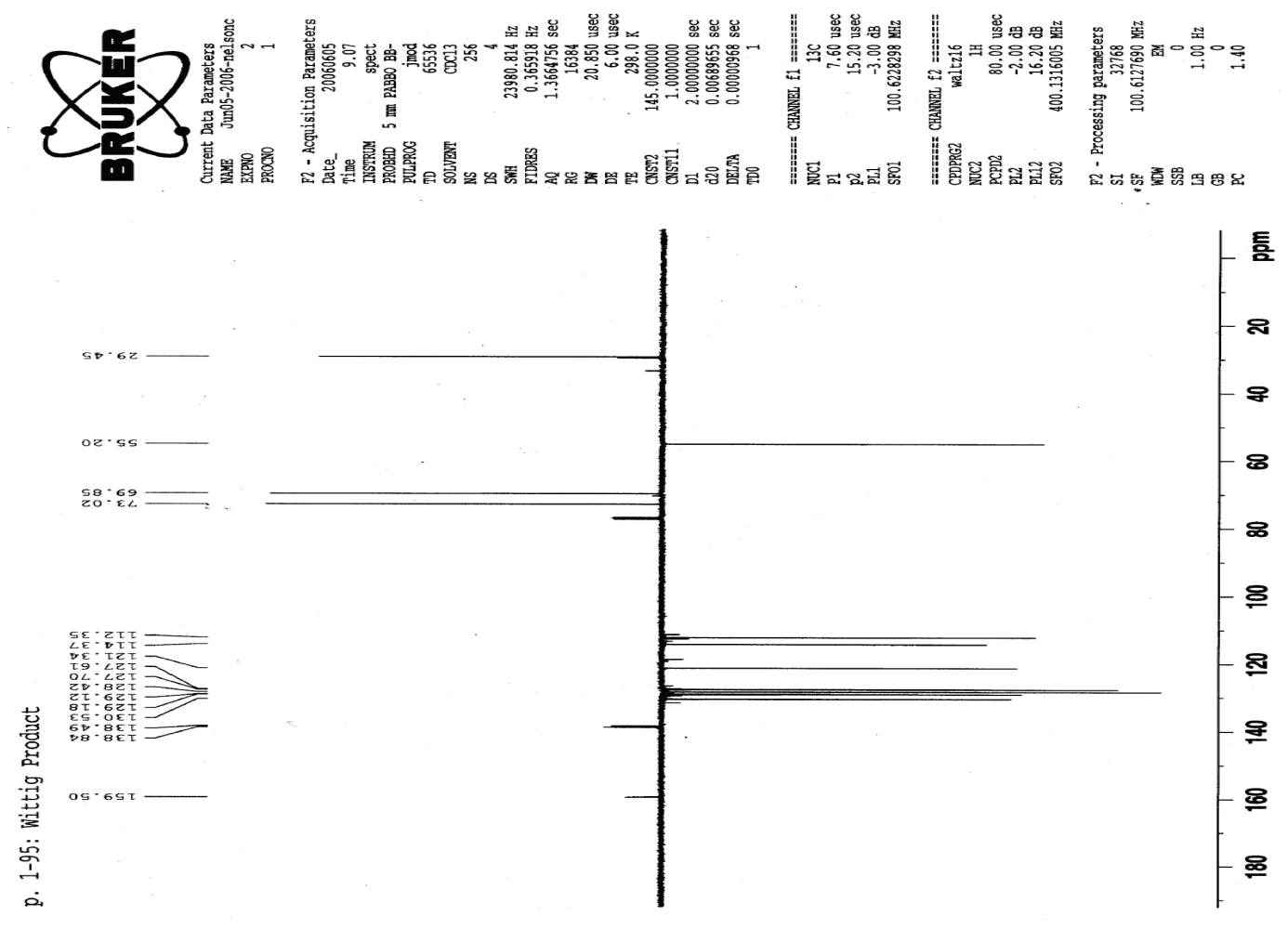

$$
\text { S-4 }
$$


${ }^{1}$ H NMR Spectrum of Alkene 8
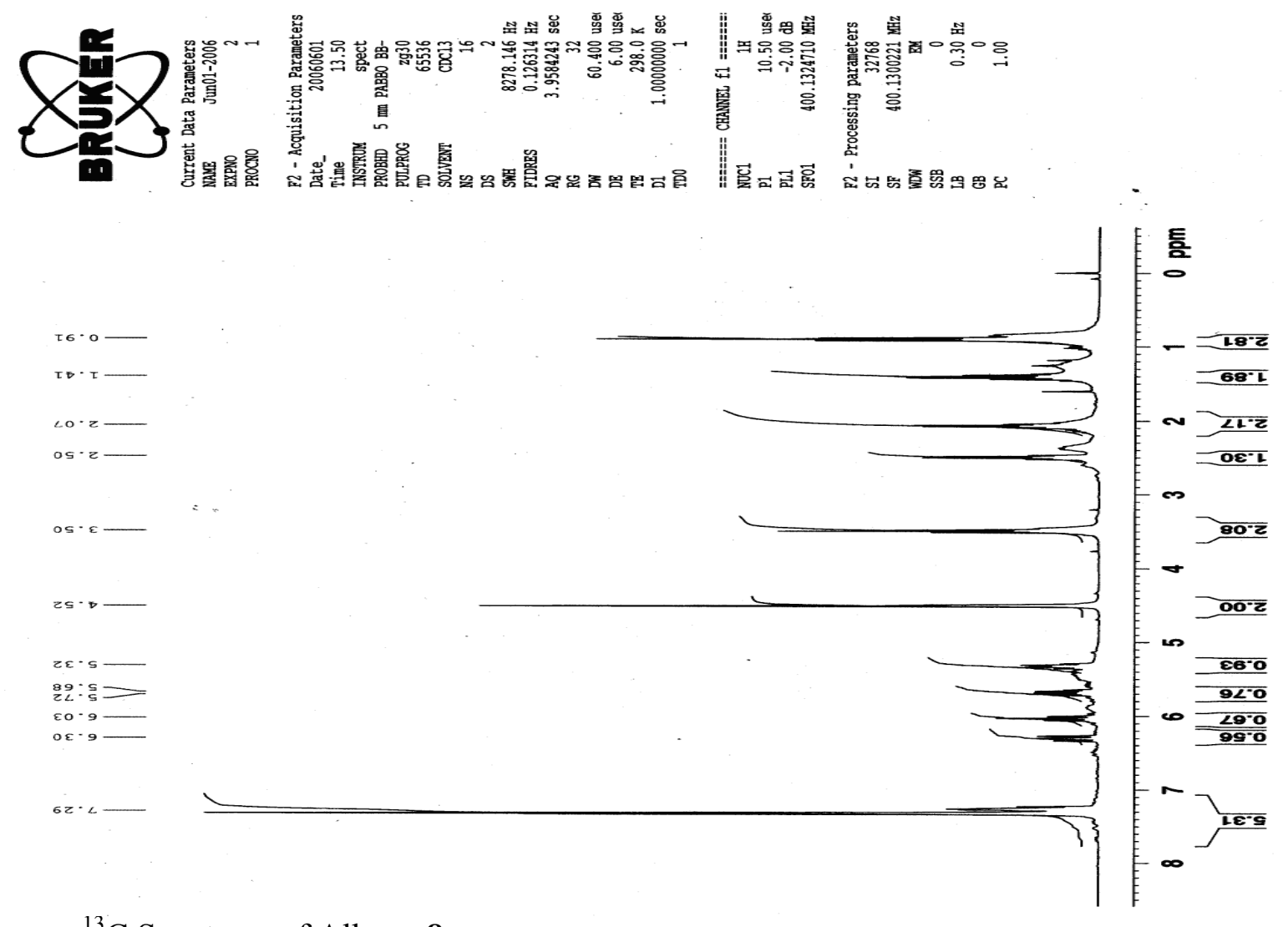

${ }^{13} \mathrm{C}$ Spectrum of Alkene $\mathbf{8}$
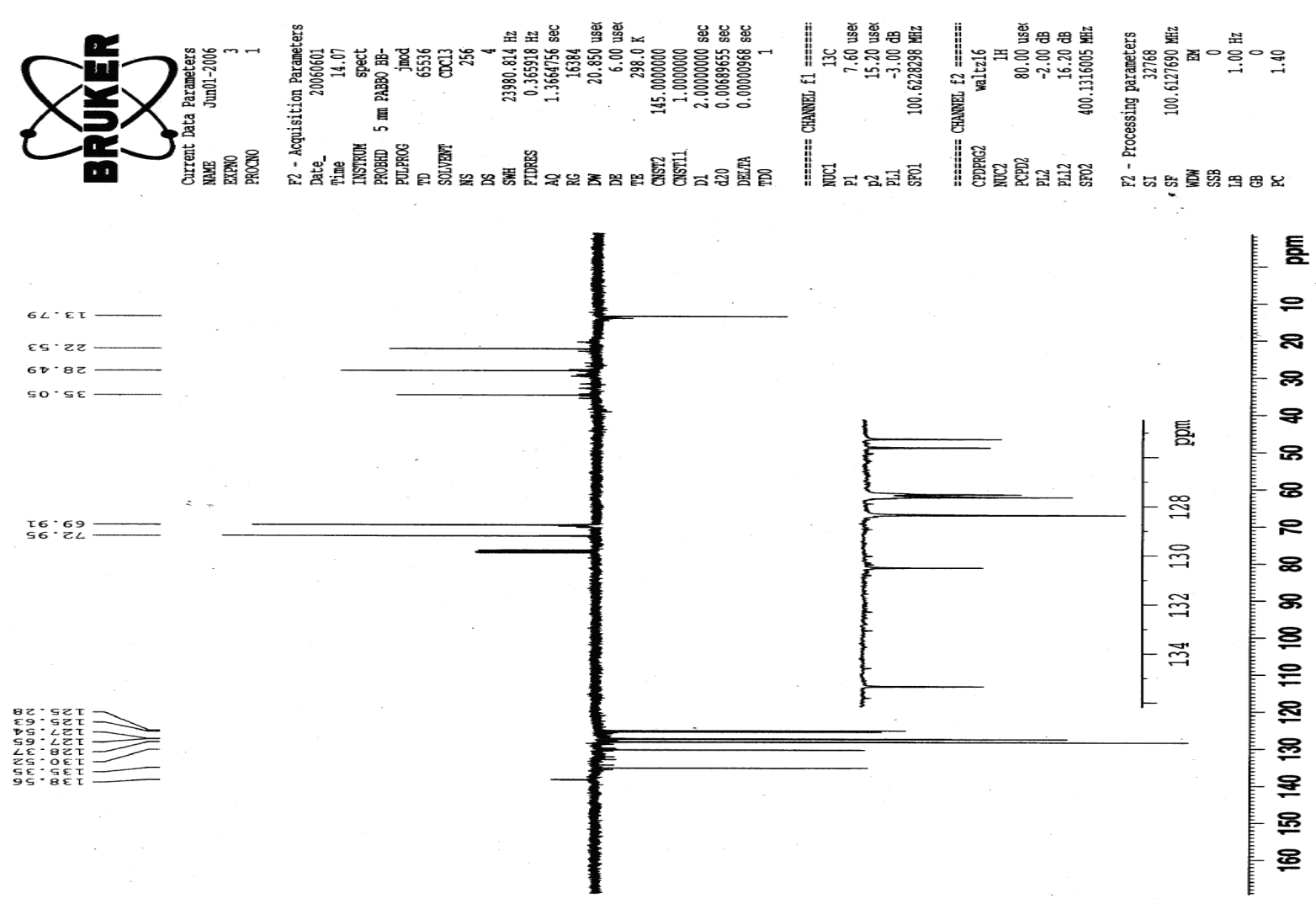

S-5 
${ }^{1} \mathrm{H}$ Spectrum of Alkene 9
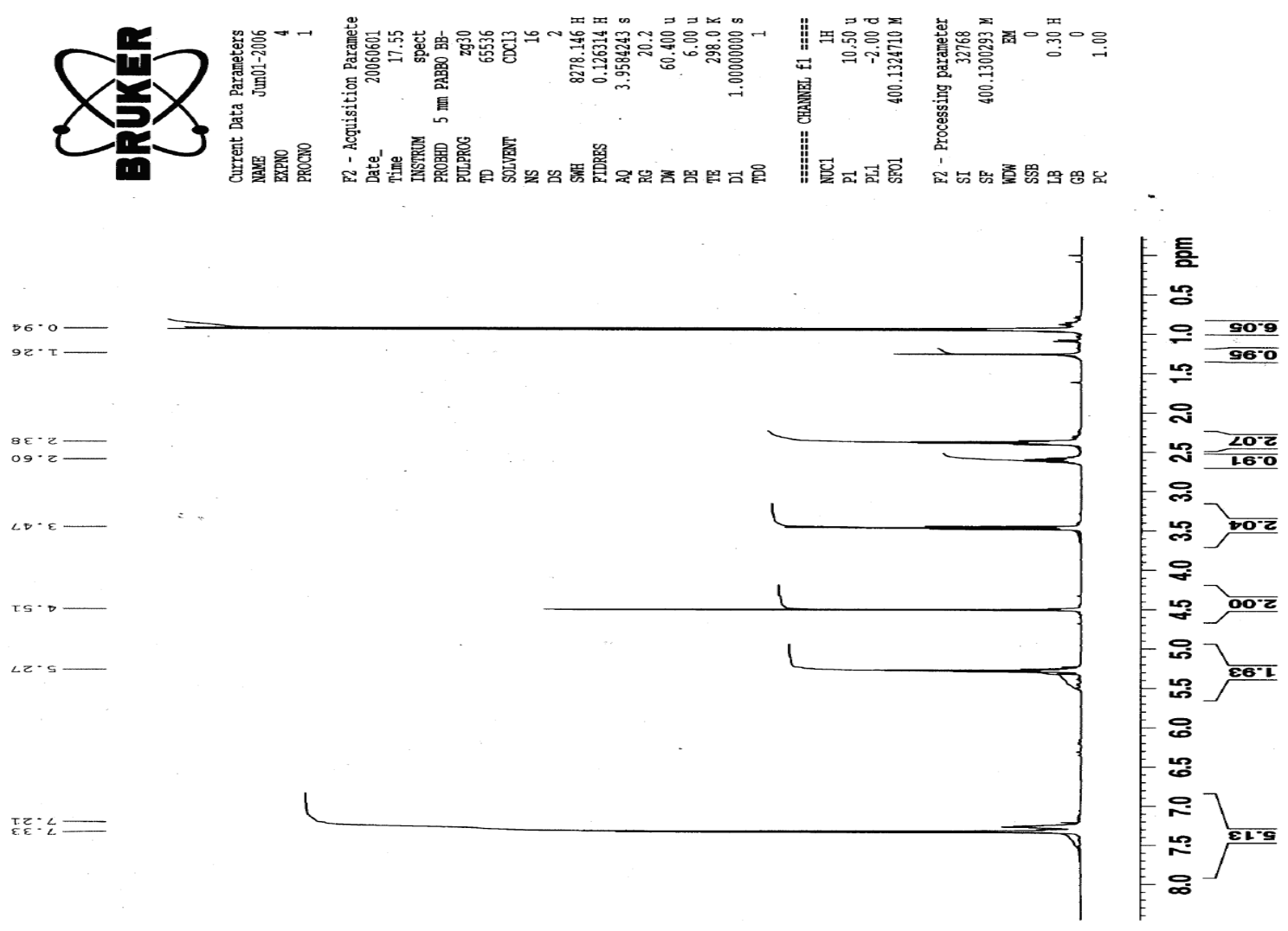

${ }^{13} \mathrm{C}$ Spectrum of Alkene 9
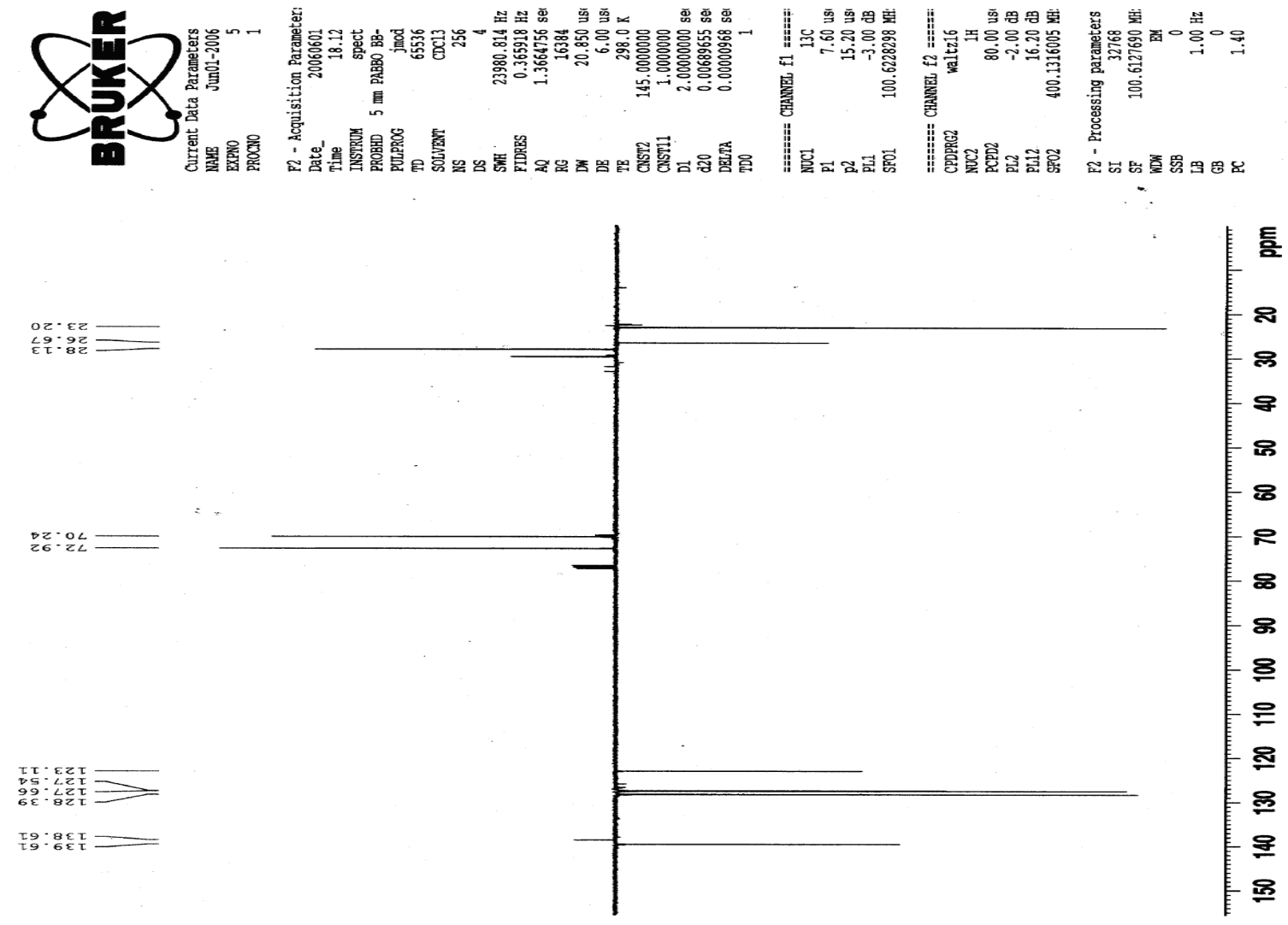
${ }^{1} \mathrm{H}$ Spectrum of Alkene $\mathbf{1 0}$
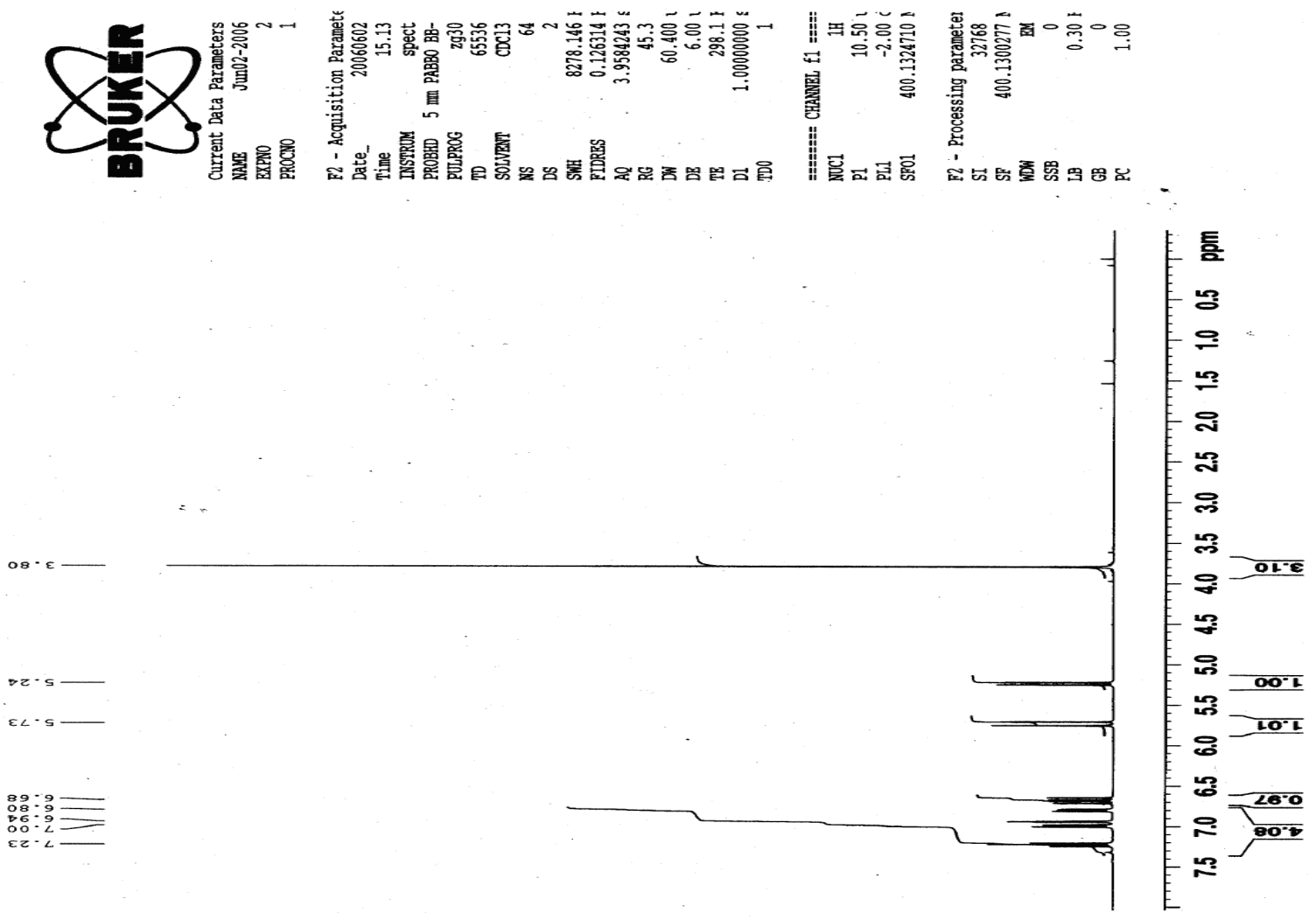

${ }^{13} \mathrm{C}$ Spectrum of Alkene $\mathbf{1 0}$
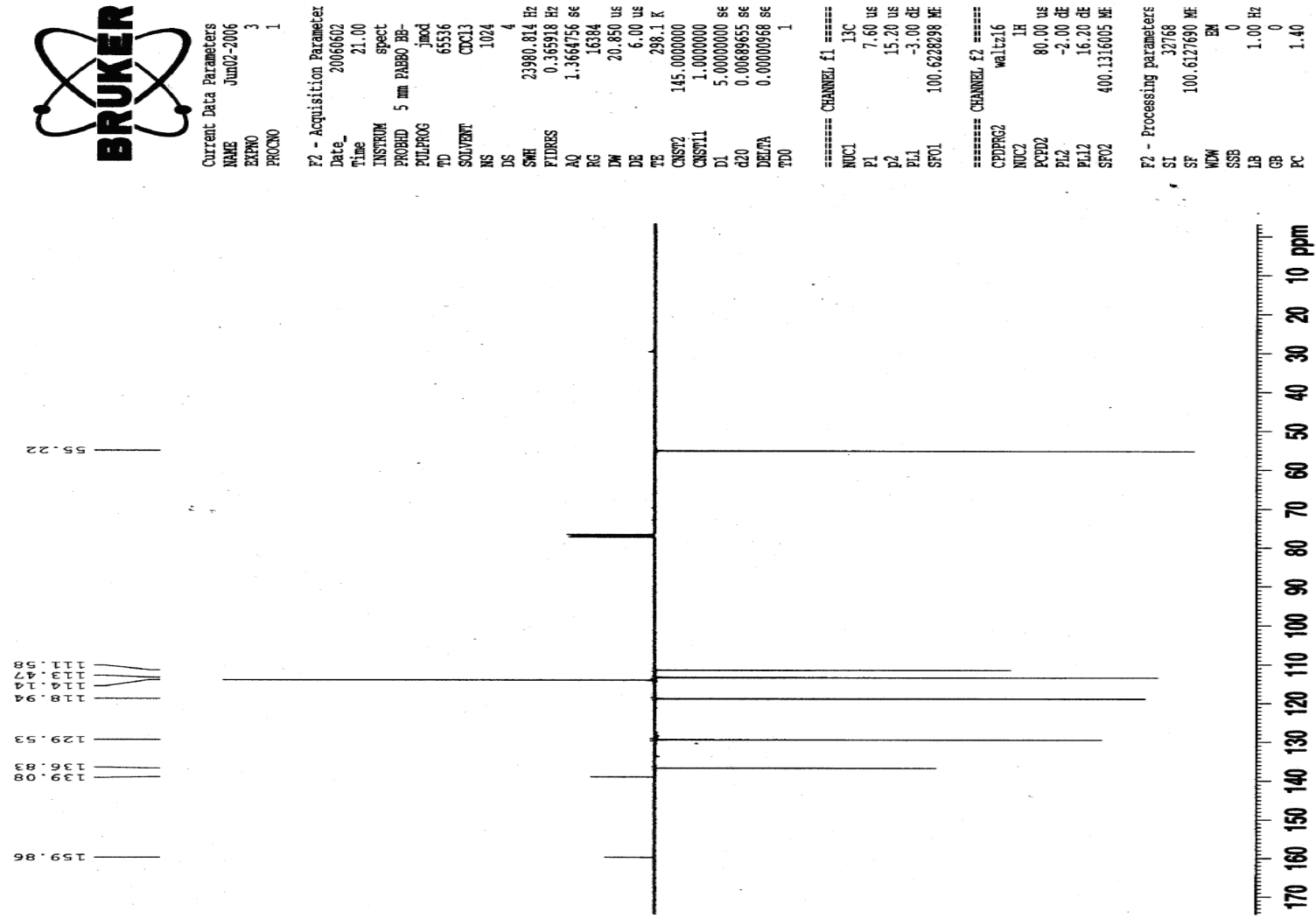
${ }^{1} \mathrm{H}$ Spectrum of Alkene $\mathbf{1 1}$
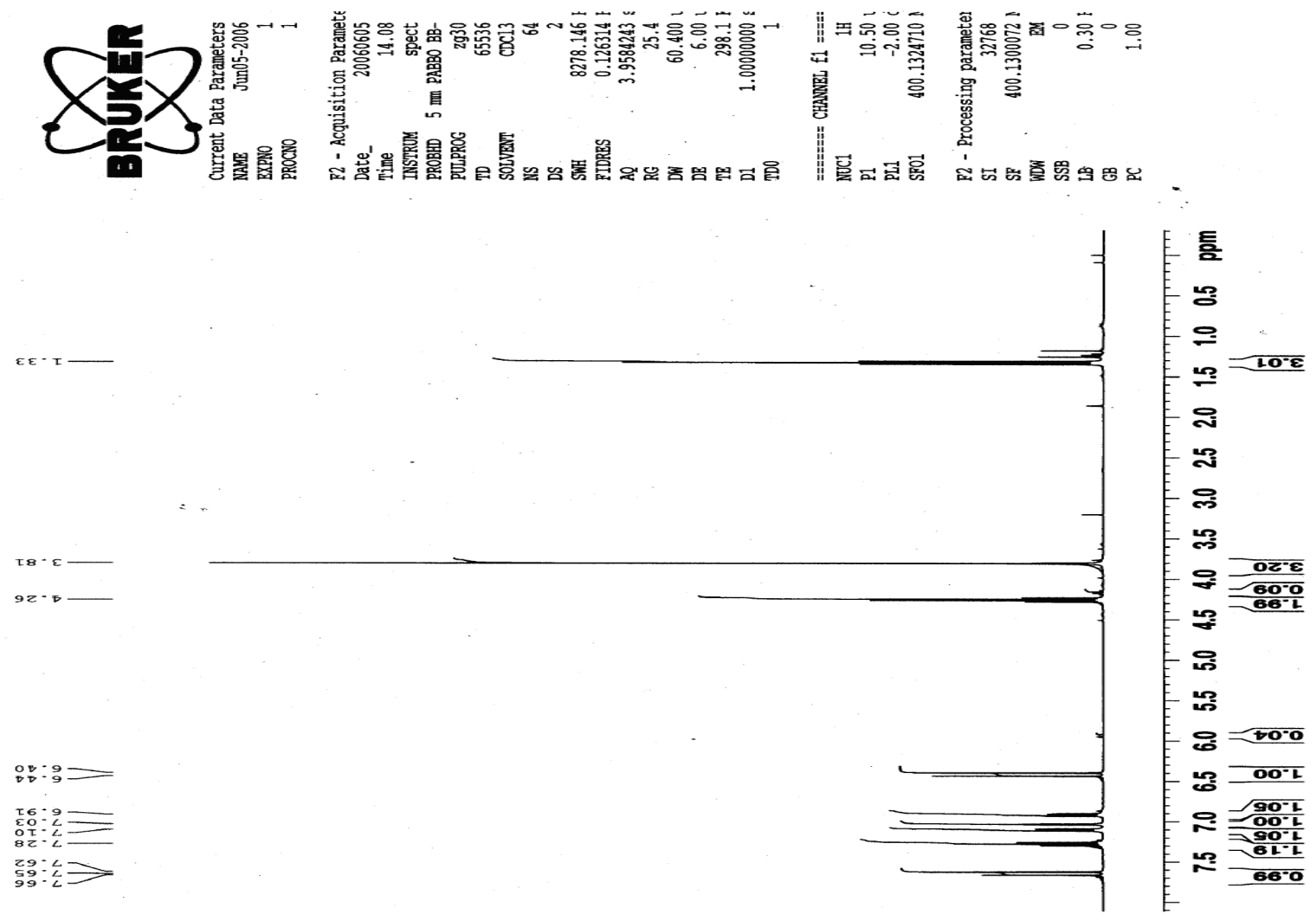

${ }^{13} \mathrm{C}$ Spectrum of Alkene $\mathbf{1 1}$
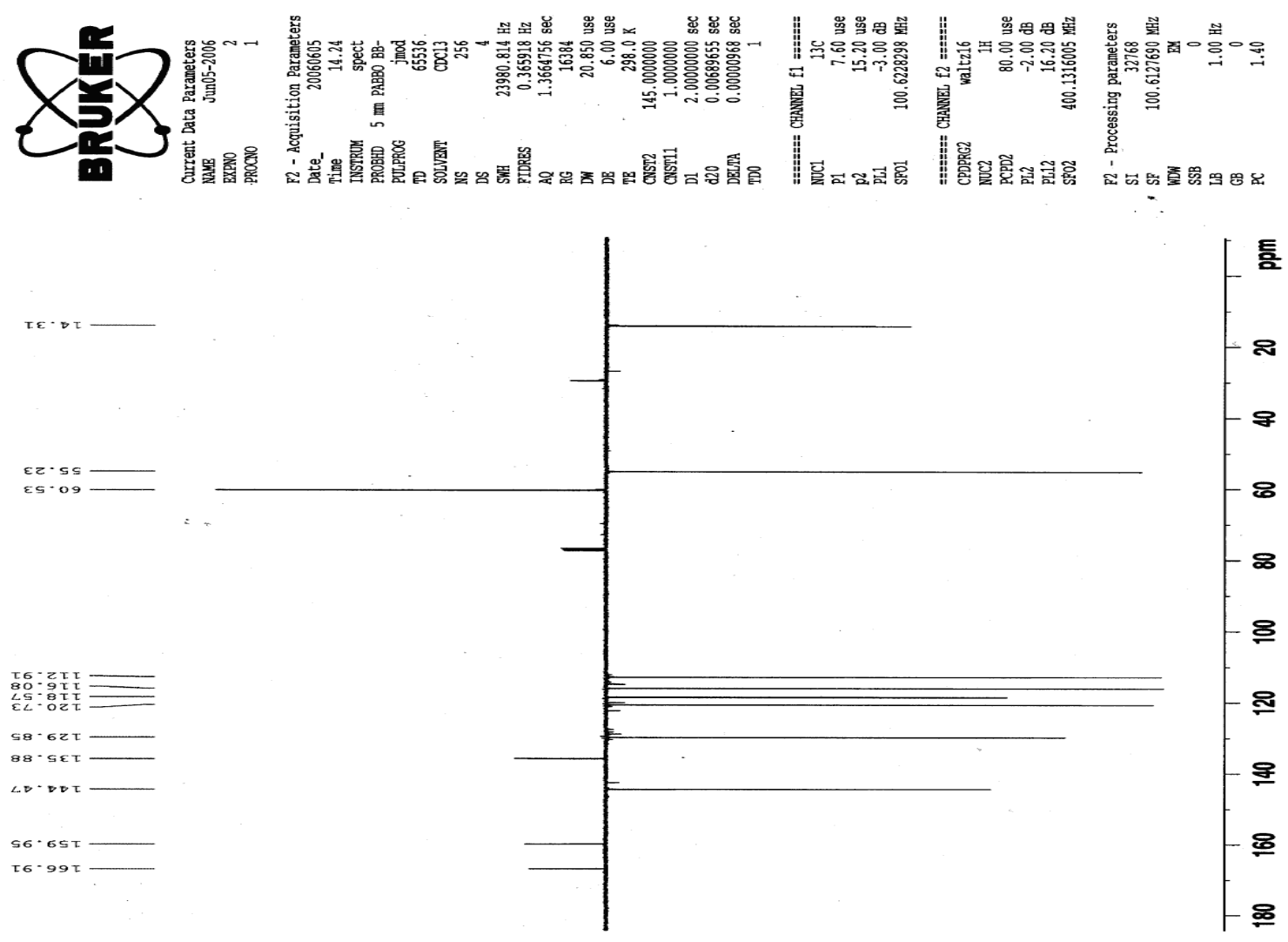